\title{
Research on the Sealing Performance of Metal Rubber Material in High Thermal Environment
}

\author{
Hui Yan ${ }^{1}$, Wei Yao ${ }^{1}$, Yalei Zhao ${ }^{1}$, Xiang $\mathrm{Bi}^{1}$, Shengbo $\mathrm{Li}^{2}$ and Hongyuan Jiang ${ }^{1, *}$ \\ ${ }^{1}$ School of Mechatronics Engineering, Harbin Institute of Technology, Harbin 150001 P.R. China \\ ${ }^{2}$ School of Mechanical and Automotive Engineering, Xiamen University of Technology, Xiamen 361024 P.R. China \\ *Corresponding author
}

\begin{abstract}
Based on the research on elastomer contact mechanics theory and percolation theory, this paper analyzes the sealing performance of metal rubber from the micro perspective of sealing coupling surface topography. By constructing the function of contacting rough surface height, the leakage model of metal rubber material seal is achieved. Comparing the leakage situation under different amplification factors, the critical amplification factor is achieved and the empirical equation to predict the leakage rate of metal rubber is given. The equation calculated the leakage rate under different working conditions and the calculated value highly fits the theoretical value, thus obtain the changing rule of the leakage according to the change of temperatures and pressures. Besides, it also provides certain reference for predicting the sealing performance of metal rubber under certain temperature and pressure.
\end{abstract}

Keywords-metal rubber material; sealing performance; temperature environment; environmental research

\section{INTRODUCTION}

Metal rubber technology is a key national defense technology which is mainly applied in harsh aerospace working conditions to solve difficult problems such as damp shock absorption, heat conduction and throttling. Achieved by a special technique, the specific interior structure of this elastic porous material decides the specialty of its application environment, for example, high vacuum, ultra-low temperature, UV radiation and corrodent ${ }^{[1]}$. Metal rubber seal is used in special working conditions, and its application would solve the sealing problems in the special environment of aviation and national defense field, and its application is highly regarded by related departments.

Sealing is to prevent the leakage of sealed medium with multiple sealing procedures. The sealing properties of metal rubber seals are similar to those of rubber seals since both of them belong to compressional sealing. As soon as metal rubber material is compressed to a groove with a certain depth, elastic deformation takes place and the reactive force of the material to the coupling surface generates a high line contact load. At the same time, deformation occurs to the coating layer material and fills the depressions of the flanges, blocking the leakage channel. In high temperature condition, metal rubber expands with the heat and could compensate for the decrease of contact stress of the coating layer after the loose in temperature. Therefore, the advantages of the sealing properties of metal rubber in high temperature are evident. On the micro level, the contact between metal rubber and antithetical flanges is a contact between two rough surfaces ${ }^{[2]}$. When no load is applied, only a few micro-bulges contact on the two surfaces of the nominal contacting area, with the increasing of the load, the contact number of the micro-bulges rises, and the contacting surfaces becomes closer, then partial plastic deformation takes place, leading to the overlapping and embedment of the microbulges with each other. In this case, the micro clearance caused by the irregularity of the surfaces is blocked, and the flow resistance to the sealing medium is increased. This combination of elasticity and plasticity enables the metal rubber to process good resilience and sealing property, which can compensate for the stress relaxation of the seal caused by the transformation of temperature and pressure.

The sealing of metal rubber material possesses the properties of high pressure resistance, corrosion resistance, radiation resistance, aging resistance, high loading capacity and wide working temperature range, which show incomparable advantages to the other sealing materials. The application of metal rubber material into the sealing under harsh working conditions has great significance to aviation, national defense weapon manufacturing, and other practical fields ${ }^{[3]}$. Currently, researches in the leakage rate of metal rubber seal are still limited, and there is few existing theory and model can be used to predicate the leakage rate of metal rubber seals. Taking the effect of the sealing surface roughness on the contact stress between the coupling surfaces into consideration, it is a difficult task to predict the theoretical leakage rate of metal rubber. This paper takes the elastomer contact area theory and the percolation theory of Persson and the theoretical basis ${ }^{[4,5]}$, and from the perspective of sealing surface roughness, this constructs a model for the metal rubber leakage rate, and deviate the corresponding empirical equation of leakage rate.

\section{The Model Building of Metal Rubber Seal LEAKAGE}

The overall model of the prediction method of metal rubber seal leakage is shown in Fig. 1. The leakage model is mainly constructed by three sub-models, which are overall finite element contact model, local finite element contact model and leakage model. Input each parameter into the leakage model could achieve the leakage rate of metal rubber seal after the calculation. The input of the parameters consists of four parts, which are, the pressure difference between the two sides of the 
metal rubber seal ring $(\Delta \mathrm{P})$, critical amplification factor $(\xi \mathrm{c})$, fluid viscosity $(\eta)$ and leakage rate correction factor $(\alpha)$.

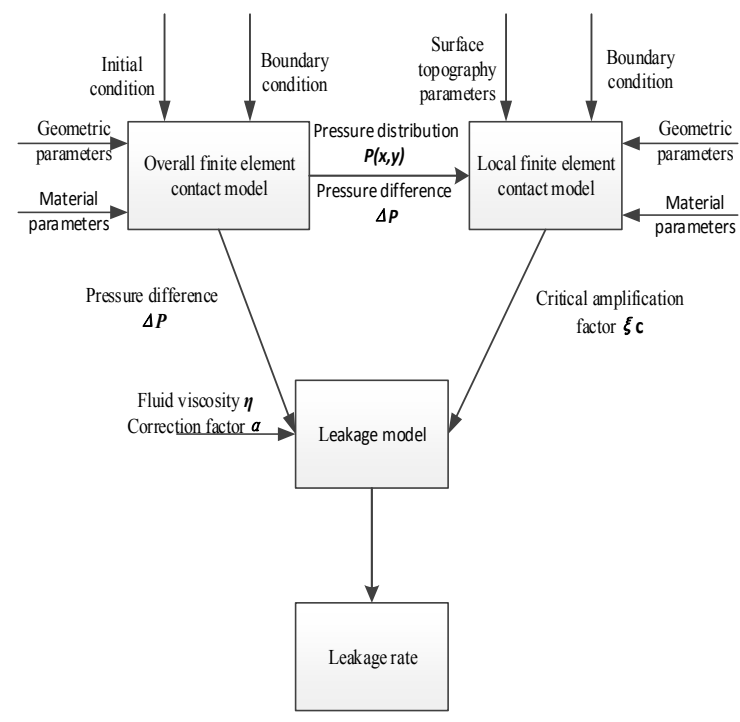

FIGURE I. OVERALL MODEL OF THE PREDICTION METHOD OF METAL RUBBER SEAL LEAKAGE

In most circumstances, rough surface is described by the altitude distribution, gradient, and angularity of the surface micro-bulges ${ }^{[6]}$. Fractal theory is perfect to describe the characteristics of the rough surface without the limit of length, and the three-dimensional rough surface can be represented by the revised two-variable Weierstrass-Mandelbrot function ${ }^{[7]}$. The presented altitude distribution function of the rough surface micro-bulges is continuous and non-differentiable between the upper and lower limited frequency, and it is independent of the length scale, besides, the generated rough surface is self-affine ${ }^{[8,9]}$. There is a strong relation between the surface topography and the necking size of the leakage channel in the following section, and it is the bridge to connect the percolation theory and the leakage rate ${ }^{[10,11]}$, moreover, selfaffinitive surface contact mechanics is the mechanical basis of them.

\section{The EQuation Derivation of MEtal RubBer LEAKAGE RATE}

The derivation of the empirical equation of metal rubber leakage rate is based on the percolation theory, and this theory is proposed by B.N.J. Persson based on the recently developed contact mechanics after accurately consideration of the elastic coupling in the contacting area between the rubber and the cemented substrates. Assume the nominal contacting area between the sealing element and the mating plate is rectangle, and the size of it is Lx $\times$ Ly (shown in Fig. 2), and assume the $\mathrm{x}>\mathrm{Lx}$ area is the low pressure side and the $\mathrm{x}<0$ side is the high pressure side of the fluidic. The rectangle area can be divided by several square areas with the length of $L x=L$, so that the area of the square is $A_{0}=L^{2}$. Here, assume $\mathrm{N}=\mathrm{Ly} / \mathrm{Lx}$ is integer, and this assumption does not affect the final effect.

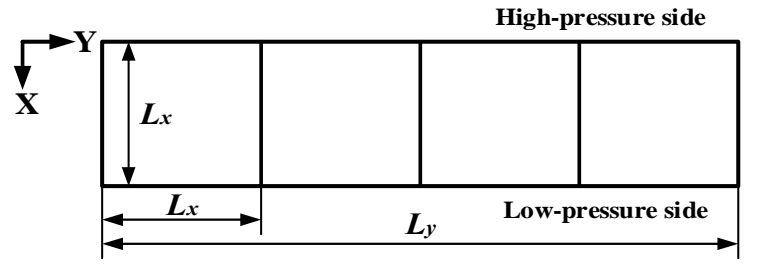

FIGURE II. THE NOMINAL CONTACTING AREA OF THE SEALING ELEMENT AND MATING PLATE SURFACE

Here, introduce in the amplification factor $\xi$, in which $\xi=L / \lambda, \lambda$ is resolution. The actual contacting area between the sealing element and the mating plate changes with the alter of $\xi$, so that $\mathrm{A}=\mathrm{A}(\xi)$. When amplification factor increases to critical amplification factor $\xi \mathrm{c}(\xi=\xi \mathrm{c})$, the leakage channel formed by non-contacting area appears for the first time. According to the Fig.2, the narrowest necking size along the leakage direction is $\lambda c=L / \xi c$, and the sealing clearance there is $u_{c}=u_{1}\left(\xi_{c}\right)$.

The differential pressure between the two sides is $\Delta \mathrm{P}$. When amplification factor reaches critical amplification factor $\xi \mathrm{c}$, the necking width $\lambda c=L / \xi c$, and the size of the clearance $u_{c}=u_{1}\left(\xi_{c}\right)$. For the incompressible Newtonian fluid, the fluid flow is laminar flow, and the fluid volume passes the necking position in unit interval can be presented as ${ }^{[12]}$ :

$$
\begin{gathered}
\dot{Q}=N M \Delta P \\
M=\alpha \frac{\mu_{1}^{3}\left(\xi_{c}\right)}{12 \eta}
\end{gathered}
$$

In this equation, $M$ is the coefficient of leakage rate, $\eta$ is the fluid viscosity $(\mathrm{Pa} \cdot s), \alpha$ is the correction factor of leakage rate. According to a large number of experiments, when the temperature of 40-120 degrees, $\alpha=0.4$; When the temperature of 40-120 degrees, $\alpha=0.2$.

To solve the leakage rate of the fluid, the necking clearance $u_{c}$ must be solved. The equation of $u_{1}(\xi)$ can be expressed as:

$$
u_{1}(\xi)=\bar{u}(\xi)+\vec{u}(\xi) A(\xi) / A^{\prime}(\xi)
$$

In this equation, $u(\xi)$ is the average clearance between the nominal contacting area when amplification factor is $\xi$. The value of it can be calculated by equation (4) ${ }^{[13]}$ :

$$
\begin{aligned}
& \bar{u}(\xi)=\sqrt{\pi} \int_{\xi q_{0}}^{q_{1}} d q q^{2} C(q) w(q) \times \\
& \int_{p(\xi)}^{\infty} d p^{\prime} \frac{1}{p^{\prime}}\left[\gamma+3(1-\gamma) P^{2}\left(q, p^{\prime}, \xi\right)\right] e^{-\left[w(q, \xi) p^{\prime} / E^{*}\right]^{2}}
\end{aligned}
$$

$C(q)$ is the power spectrum of surface roughness, and its expression is: 


$$
C(q)=\frac{1}{(2 \pi)^{2}} \int\langle z(x) z(0)\rangle e^{-i q \cdot x} d^{2} x
$$

According to the percolation theory, under different amplification factors, the normal contact pressure of metal rubber cemented substrates is static, so that:

$$
p(\xi)=P_{0} A_{0} / A(\xi)
$$

In which, $P_{0}$ is the nominal contact pressure $(\mathrm{MPa})$ when amplification factor is $1, A_{0}$ is the nominal contact area $\left(\mathrm{mm}^{2}\right)$ when amplification factor is 1

Besides:

$$
w(q, \xi)=\left(\pi \int_{\xi q_{0}}^{q} d q^{\prime} q^{\prime 3} C\left(q^{\prime}\right)\right)^{-1 / 2} .
$$

In this equation, $q_{0}$ is the wave vector $\left(\mu \mathrm{m}^{-1}\right)$.

The expression of $P(q, p, \xi)$ is as follows:

$$
P(q, p, \xi)=\frac{2}{\sqrt{\pi}} \int_{0}^{s(q, \xi) p} d x e^{-x^{2}}
$$

In this equation, $s(q, \xi)=w(q, \xi) / E^{*}, \quad E^{*}$ is composite elasticity modulus whose value can be derived, and it is not down in this paper. According to the above analysis, as long as $\xi c, \eta, \Delta P$ and $\alpha$ are inputted, the leakage rate of metal rubber seal can be solved.

\section{LEAKAGE RATE CALCULATION INSTANCE}

\section{A. The Determination of Critical Amplification Factor}

Scan the surface topography of the mating plate and use length scale $r_{i}(i=1 \ldots n)$ to measure the surface profile curve of flange plate by dividing, and the data meet:

$$
\lg N=-D \lg r+C
$$

Takes $n$ groups of dot pair $\left[\mathrm{r}_{1}, \mathrm{~N}_{1}\right],\left[\mathrm{r}_{2}, \mathrm{~N}_{2}\right], \ldots\left[\mathrm{r}_{\mathrm{n}}, \mathrm{N}_{\mathrm{n}}\right]$, and match these pair dots into a straight line in the double logarithmic coordinate system, and the slope of this line is the fractal dimension of the profile curve. In the actual solving process, according to the sizing theory, circle with 10 different radius can be adopted. Firstly, set the center of the circle at the beginning of the curve, and then set the intersection point of the circle and the curve as the beginning point and looks for the next intersection point. Go on this procedure until the whole curve is measured. The straight line matched by the dot pairs is shown in Figure 3.

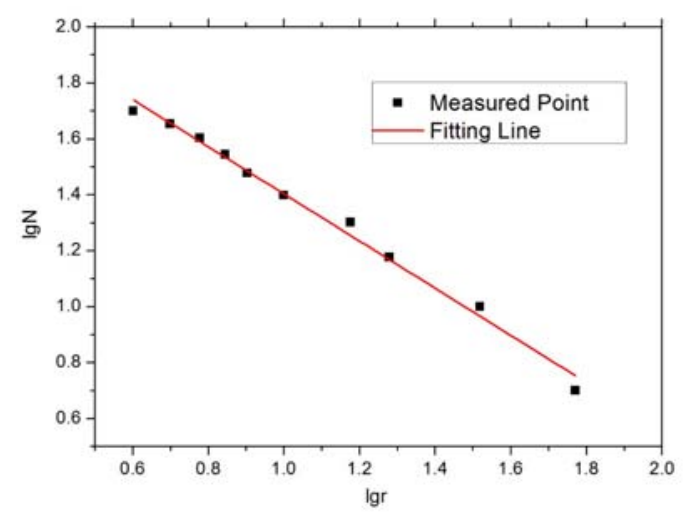

FIGURE III. THE MATCHING OF THE PAIR DOTS IN DOUBLE LOGARITHMIC COORDINATE SYSTEM

The fractal dimension of the surface profile of the flange plate $\mathrm{D}=1.18$, thus the fractal dimension of the flange plate $\mathrm{DS}=2.18$. Based on the relation between fractal dimension and Hurst index, it can be conducted that $\mathrm{H}=0.82$.

Making use of the local finite element model, it can be concluded that the contacting area between the metal rubber seal and the mating plate decreases with the increasing of amplification factor under the effect of normal uniform load. Because the bigger amplification factor is, the more microbulges would be on the rough surface, so that it would be harder for the two surfaces to be sealed with each other. When amplification factor is raised to $\xi=12.3$, non-contacting area continues to amplify, and at the same time, it can be observed that under the effect of certain uniform load, leakage channel appears for the first time, thus the critical amplification factor is $\xi \mathrm{c}=12.3$.

\section{B. The Calculation of Leakage Rate under Different Temperatures and Pressures}

Take metal rubber with the relative density of 0.40 as the example. The known parameters are as follows: the size of the seal is $135.13 \mathrm{~mm}$ (inner diameter) $\times 3.0 \mathrm{~mm}$ (cross-sectional diameter), the compression ratio is $20 \%$; the size of the flange plate: the width of the grove is $4.1 \mathrm{~mm}$, the height is $2.4 \mathrm{~mm}$, the external diameter is $141.4 \mathrm{~mm}$, the material is $1 \mathrm{Cr} 18 \mathrm{Ni}$ Ti, whose elasticity modulus $\mathrm{E}_{2}=170 \mathrm{GPa}$, poisson ratio $\mathrm{v}_{2}=0.3$, $\mathrm{E}^{*}=10.828 \mathrm{Mpa}$. Measured by surface roughness profile instrument, $\mathrm{Ra}=1.8061 \mu \mathrm{m}, q_{0}=1.0 \times 104 \mathrm{~m}^{-1}, q_{1}=7.8 \times 109 \mathrm{~m}^{-1}$, and after calculation $\mathrm{H}=0.82, \mathrm{C}_{0}=1.712, \mathrm{~A}_{0}=1538.868 \mathrm{~mm}^{2}$, The viscosity of Hydraulic oil $\eta$ is solved as follows:

(1)The viscosity of Hydraulic oil $\eta$.

The hydraulic oil used in the experiment is Total fireproofing hydraulic oil (type number: TOTAL HYDRANSAFE HFDU 46). This hydraulic oil is biodegradable oil, which is free from hazardous substances. Besides, it processes outstanding lubricating property and high ignition point as well as autoignition temperature. The property parameters of it mainly includes: the kinematic viscosity in $40^{\circ} \mathrm{Cand} 100^{\circ} \mathrm{C}$ is $47.5 \mathrm{cSt}$ and $9.35 \mathrm{cSt}$ respectively, the viscosity index is 185 , ignition point $358^{\circ} \mathrm{C}$ and the autoignition 
temperature is higher than $420^{\circ} \mathrm{C}$. Then the dynamic viscosity in $100^{\circ} \mathrm{C}$ is as follows.

$$
\eta=\rho v=0.00864875 \mathrm{~Pa} \cdot \mathrm{S}
$$

When the pressure of mineral oil is less than $20 \mathrm{MPa}$, the pressure has minor effect on viscosity. The experimental pressures in his paper are all within $20 \mathrm{MPa}$, and the Reynolds viscosity-temperature equation is presented as equation (11).

$$
\eta=\eta_{0} e^{-\beta\left(T-T_{0}\right)}
$$

In the equation, $\eta$ is the viscosity $(\mathrm{Pa} \cdot \mathrm{s})$ when temperature is $\mathrm{T}, \eta_{0}$ is the viscosity ( $\left.\mathrm{Pa} \cdot \mathrm{s}\right)$ when temperature is $\mathrm{T}_{0}, \beta$ is viscosity-temperature coefficient $\left(0.03 /{ }^{\circ} \mathrm{C}\right)$

(2) Substitute the above data into equation (1), and calculate the theoretical and experimental value of leakage rate under each working temperature. The results are as follows.

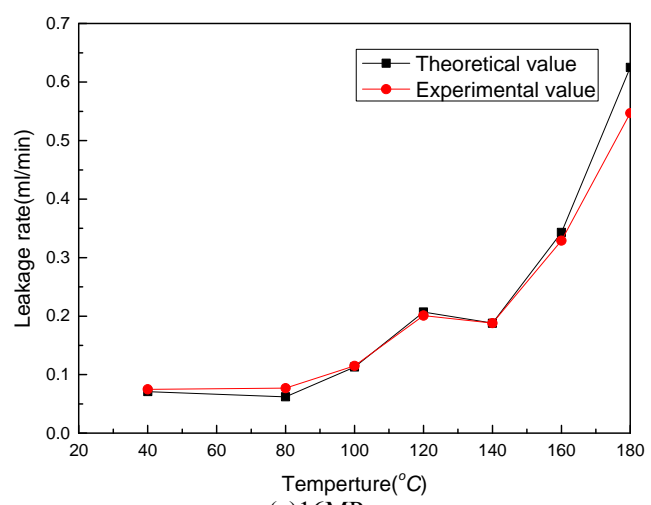

(a) $16 \mathrm{MPa}$

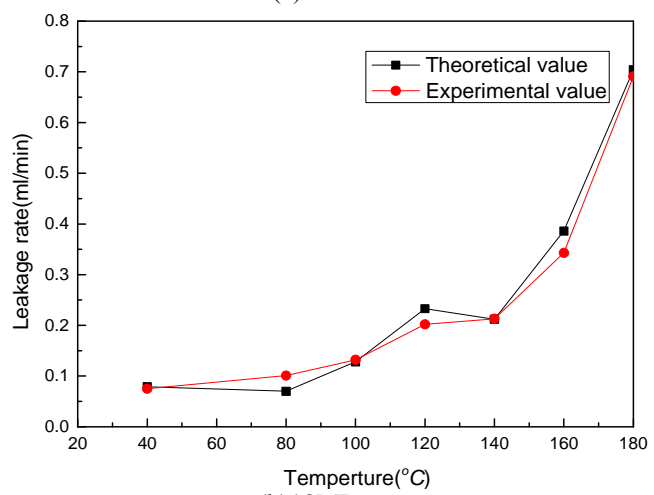

(b) $18 \mathrm{MPa}$

FIGURE IV. THE COMPARISON OF THE THEORETICAL VALUE AND EXPERIMENTAL VALUE UNDER DIFFERENT PRESSURES

It can be seen from the comparison between the theoretical and experimental values that within the temperature range from 40 to $180^{\circ} \mathrm{C}$, the theoretical values approximately agree with the experimental values. The existed errors are mainly caused by certain simplifications of secondary factors during the calculation process. Besides, the errors are also caused by systematic errors in the applications of the experiment. The above analysis indicates that the leakage rate equation conducted in this paper can be used in the analyzation of the leakage rate of metal rubber seal in low temperature condition.

\section{CONClusions AND PERSPECTIVES}

This paper takes the elastomer contact mechanics theory and percolation theory of Persson as the research basis, and in combination of the micro sealing performance of metal rubber, this paper constructs the prediction leakage model from the micro perspective of the coupling contacting urface topography between the metal rubber sea and the mating plate. On the one hand, this paper proposes a modeling approach based finite element thought and conducted the empirical equation of leakage rate. On the other hand, for the sealing of metal rubber material with specific parameters, this paper also provides the simulation result and the experimental equation for its leakage based on specific experimental working condition. The calculated values accords well with the empirical equation, so that this paper provides a basis for predicting the sealing performance of metal rubber material under certain temperature and pressure.

\section{ACKNOWLEDGMENT}

The authors thank the anonymous reviewers for their critical and constructive review of the manuscript. This study was supported by the National Natural Science Foundation of China (No:51405410) and the Air Force Armament Department Pre-Research Foundation.

\section{REFERENCES}

[1] Yan H, Zhang W J, Jiang H Y and Chen L 2014 Chin. Phys. B 23 040702

[2] Cui Xiaojie 2011 China Petroleum Machinery 39(C00): 102-105.

[3] Zhang W G, Lin G M, Shang M, et al 2014 Advanced Materials Research 912:810-813.

[4] Persson B N J, Albohr O, Creton C, et al 2004 The Journal of chemical physics 120(18): 8779-8793.

[5] Lorenz B, Persson B N J 2009 EPL (Europhysics Letters) 86(4): 44006.

[6] Sahoo P, Ghosh N 2007 Journal of Physics D: Applied Physics 40(14): 4245.

[7] Yan W, Komvopoulos K 1998 Journal of Applied Physics 84(7): 36173624 .

[8] Ausloos M, Bermon D 1985 Proceedings of the Royal Society of London A Mathematical and Physical Sciences 400(1819): 331-350.

[9] Blackmore D, Zhou J G 1998 . Fractals 6(01): 43-58.

[10] Dapp W B, L Cke A, Persson B N J, et al 2012 Physical review letters 108(24): 244301.

[11] Persson B N J, Prodanov N, Krick B, et al 2012 The European Physical Journal E: Soft Matter and Biological Physics 35(1): 1-17.

[12] Persson B N J, Yang C 2008 Journal of Physics: Condensed Matter 20(31): 315011 .

[13] Yang C, Persson B N J 2008 Journal of Physics: Condensed Matter 20(21): 215214. 